\title{
Automated decision-making system based on genetic algorithm in managing maintenance process
}

\author{
Aleksey Nikishenko ${ }^{1, *}$, Elena Boyko ${ }^{2}$, and Yuriy Obzherin ${ }^{2}$ \\ ${ }^{1}$ KB KA Ltd., 299003 Sevastopol st. Revolution 1905, 12,, Russia \\ ${ }^{2}$ Sevastopol state University, Department of higher mathematics, 299053 Sevastopol st. Universitetskay, 33, Russia
}

\begin{abstract}
An automated decision-making system in managing the maintenance process was developed. The problem of vector optimization was set. A genetic algorithm with domination sorting and elite selection was used to find a multitude close to the Pareto set in multi-criteria optimization.
\end{abstract}

\section{Introduction}

The choice of strategy and frequency of monitoring, as well as the maintenance method refers to solutions that have a serious long-term impact. Therefore, strategic decisions of this kind deserve careful analysis, including mathematical modeling, and, most importantly, in the face of constantly changing factors (the quality of components, the cost of modules and materials, the size of the expected profit) require the creation of an automated decision-making system (ADMS). The structure of the ADMS was developed taking into account modern requirements for such systems and analysis of existing standard structures. Its peculiarity is division into mutually independent and interacting parts. The "decision making" is a specific function of the ADMS, which distinguishes it from automated computer (information) systems. An analysis of various literature sources [1-3] showed that genetic algorithms (GA) show good applicability for solving optimization problems.

\section{Structure of ADMS in managing maintenance process}

ADMS in managing maintenance process is a multi-level system designed to organize and manage the maintenance process of the production system (PS), which is based on the assessment of the controlled object state during operation (the technical condition monitoring - TCM). ADMS has to take into account problematic production situations, as well as material and financial costs.

It should be noted that the maintenance process includes:

- the TCM process of the PS or its components;

- the maintenance process itself.

In general, the ADMS consists of three blocks. The first block is a block for collecting, processing and storing of information. It is the most voluminous and labor-intensive, in terms of organization. This block is not directly related to the decision-making process, but it provides the necessary base for making these or that decisions. The second block is an information system whose function is to make the prepared information foreseeable and suitable for processing. The third block is the decision support system (DSS). This block works directly with the solutions and allows to choose a specific solution based on the embedded algorithm or in the process of interaction with the decision maker (DM).

The "decision making" function is designed to find the area of optimal solutions for multi-criteria optimization or to choose the optimal solution to the problem situation associated with the malfunction of the equipment. To implement this function, the ADMS includes a set of calculation and decision-making models obtained using the theory of semi-Markov processes with a common phase space of states [4] and GA, as well as a database creation and maintenance system that provides them with information.

The general concept of building the ADMS is based on a hierarchical principle that provides the distribution of management functions between the interrelated structural levels: information, strategic, tactical and executive. The hierarchical structure of the ADMS in managing maintenance process of the PS is shown in Figure 1. The hierarchical organization of the ADMS ensures collection of data about the controlled object at the information level, choice of distribution functions and methods of monitoring at the strategic level, formation of quality criteria for PS functioning and their quantitative assessments at the tactical level, as well as management of PS maintenance process in the process of PS functioning at the executive level. Moreover, an operator controls its functioning.

The input of the system receives information from the DM and from the database. At the output of such system it is possible to obtain the following information: the efficient control frequency for the selected mode, the efficient tactics of maintenance, the technical and

\footnotetext{
* Corresponding author: sklibur@rambler.ru
} 
economic performance indicators of the PS for the selected mode.

ADMS is implemented in the form of a software system, the structural diagram of which is presented in Figure 2. It contains five main modules and includes a large number of algorithms combined into computational models that allow solving various technical problems. DSS modules, as the most complex in the organization, will be considered in more detail.

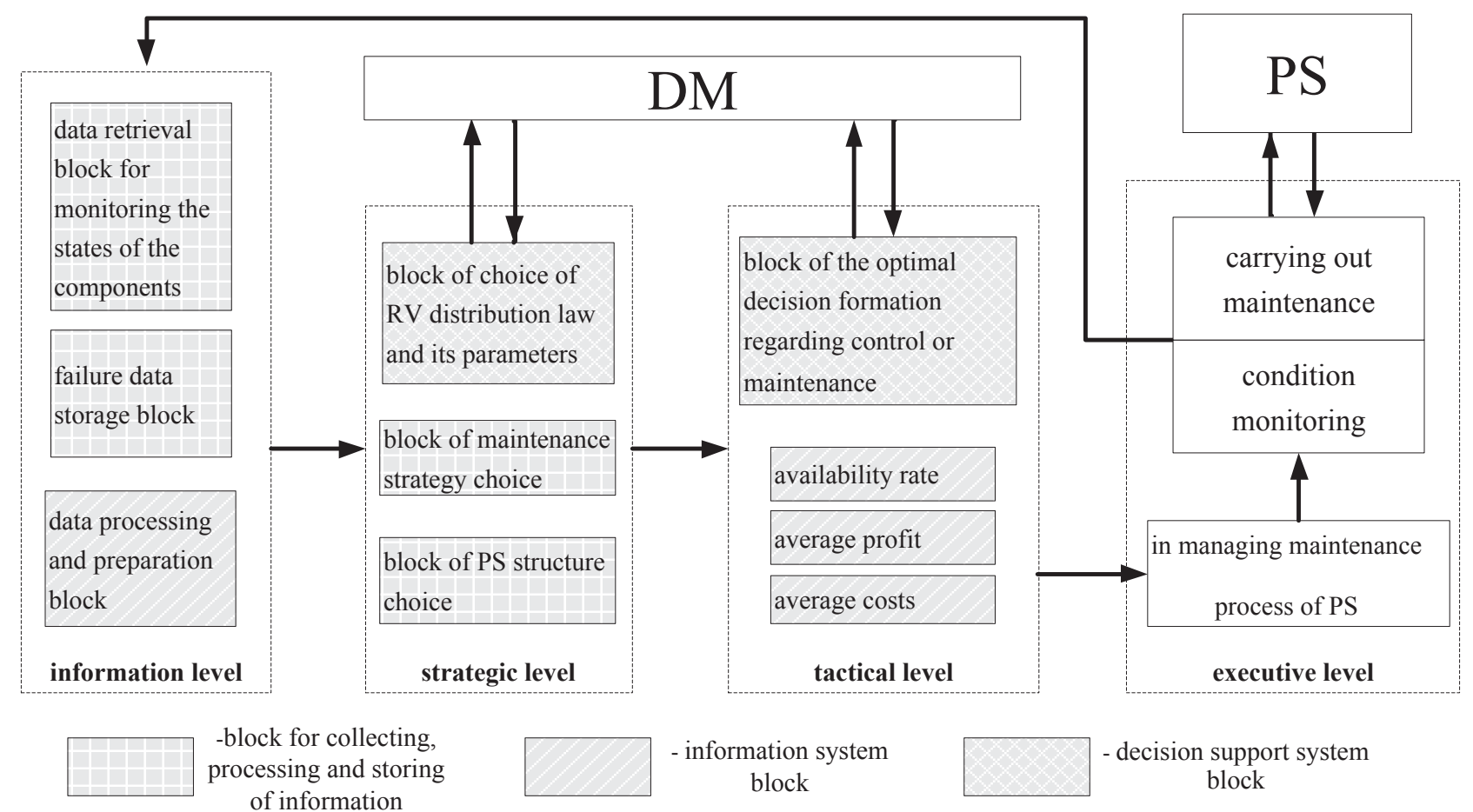

Fig. 1. Structural diagram of ADMS in managing maintenance process.

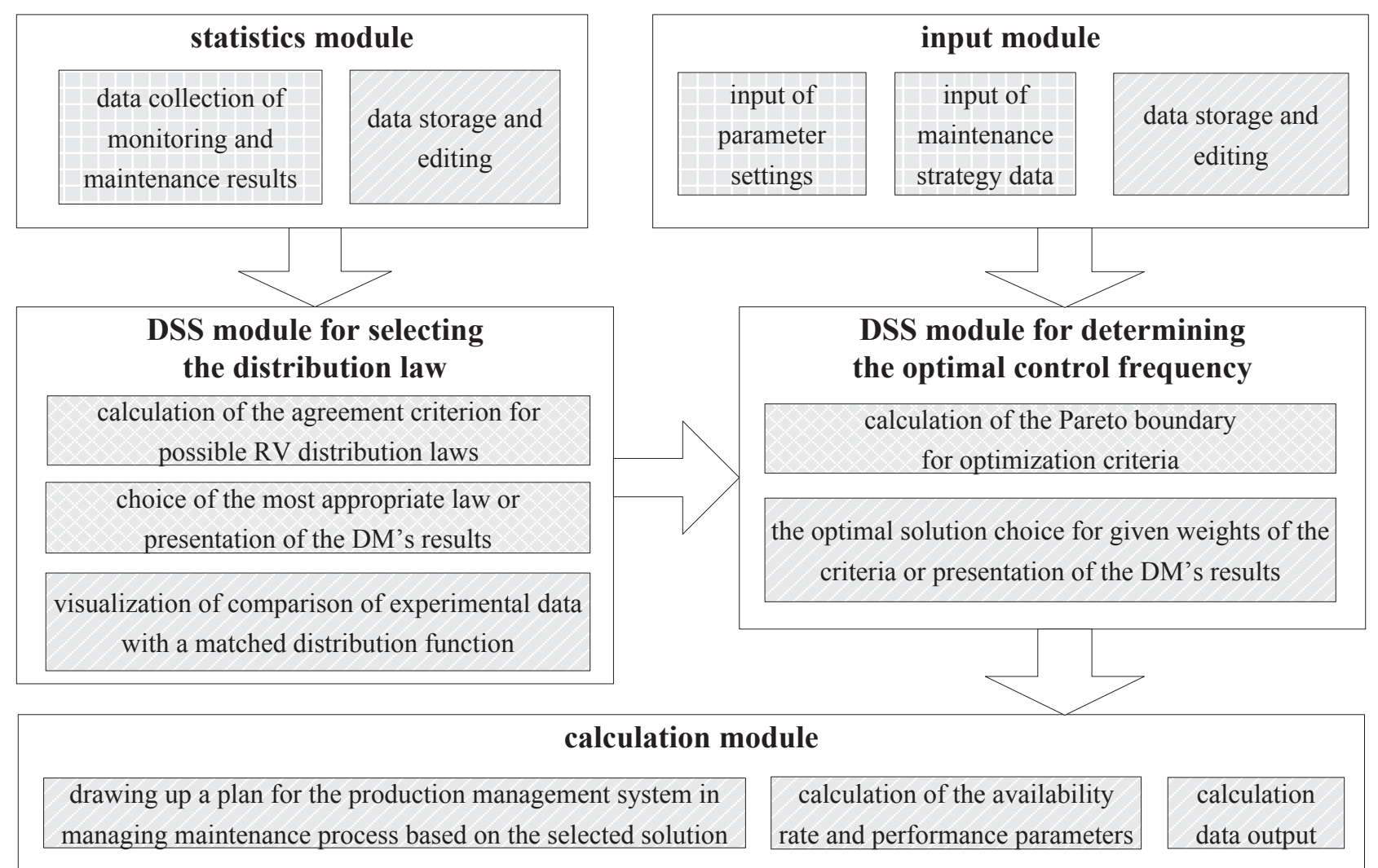

Fig. 2. Structural diagram of ADMS in managing maintenance process. 


\subsection{DSS module for choice of the random variable distribution law and/or its parameters}

The DSS module makes it possible to determine the law of random variables (RV) and/or its parameters based on the results of monitoring the presence of latent failures (LF) [5].

At the input of the DSS block, the vector of experimental values of the RV is fed by the choice of the distribution law. Before the processing, the vector is sorted and filtered (foreign objects, for example, symbols are deleted). Verification of the hypothesis about the supposed law of RV distribution is carried out in accordance with Pearson's agreement criterion (criterion $\chi^{2}$ ) [6], [7].

The following laws are used as the supposed laws of the failure times distribution in the module by default [8]: exponential distribution; Weibull-Gnedenko distribution; gamma distribution; normal distribution; lognormal distribution; uniform distribution. This list can be supplemented with user functions.

\subsection{DSS module for selection of the optimal management process}

The decision to choose a tactic of PS management is based on solving the optimization task of selecting the most effective frequency for PS monitoring.

The operation efficiency is the degree to which the actual result of the operation corresponds to the required one [9]. In practical research of effectiveness, the problem of assessing effectiveness and the problem of choosing a rational mode of action (choice of tactics) are usually singled out. The assessment of effectiveness involves formation of the purpose of the operation, the choice of the effectiveness criterion, which quantitatively expresses the degree to which the actual result corresponds to the required one.

\section{Optimization of automated control frequency for multi-component production systems with latent failures}

For the system under consideration, the reliability and economic parameters of the wave soldering production unit used to describe PS with TCM and maintenance in scientific works [10-12] are implemented as optimality criteria:

1) Stationary availability:

$$
\phi_{1}(\bar{\tau})=K_{\Gamma}(\bar{\tau}) \rightarrow \max _{\bar{\tau} \in \mathbf{T}} ;
$$

2) Average profit per unit of calendar time:

$$
\phi_{2}(\bar{\tau})=S(\bar{\tau}) \rightarrow \max _{\bar{\tau} \in \mathbf{T}} ;
$$

3) Average costs per unit of time of the efficient system functioning (with a minus sign):

$$
\phi_{3}(\bar{\tau})=-C(\bar{\tau}) \rightarrow \max _{\bar{\tau} \in \mathbf{T}} .
$$

Here $\bar{\tau}=\left(\tau_{1}, \ldots, \tau_{N}\right)-$ is the vector of the variable parameters of the problem, the admissible values region of the vector $\bar{\tau}$ forms a multitude $\mathbf{T}$.
We set the three-criteria optimization problem [1] for the management process of component-wise control: to find the values of the variable parameters $\tau_{i}, i=\overline{1,3}$, which, in the limit, ensure the fulfillment of all conditions $(1)-(3)$. The vector optimality criterion $\left(\phi_{1}(\bar{\tau}), \phi_{2}(\bar{\tau}), \phi_{3}(\bar{\tau})\right)$ is defined on the multitude $\mathbf{T}$, and the DM strives to maximize each value of the particular optimality criteria $\phi_{i}(\bar{\tau}), i=\overline{1,3}$ :

$$
\max _{\bar{\tau} \in \mathbf{T}}\left(\phi_{1}(\bar{\tau}), \phi_{2}(\bar{\tau}), \phi_{3}(\bar{\tau})\right)
$$

Usually, the optimality criteria are contradictory, and they reach their optimal values at various points in the multitude of admissible values of the variable parameters vector T. Therefore, a preliminary solution to the problem is a multitude of compromise solutions, called the Pareto set. This multitude includes solutions that cannot be improved immediately by all the criteria.

\subsection{Multi-criteria optimization using genetic algorithm for finding solutions}

For the system under study, there are three system performance criteria, which depend on three control parameters (on $\mathrm{N}$ parameters for the $\mathrm{N}$-component system). In the optimization process, it is necessary to determine the control parameters values that will ensure the efficiency of the system operation.

This task has the following peculiarities:

- multi-criteria;

- a large number of alternative solutions;

- the objective function can have many local extremums in view of consideration of the general form of the $\mathrm{RV}$ distribution law.

It is obvious that a simple enumeration of possibilities can take too much time. Thus, there is a problem of finding within the acceptable time the optimal or close to the optimal solution.

The solving of problems with the above-mentioned features makes it possible to simplify the usage of GAs, which are used to determine the frequency of TCM installation blocks close to the optimum from the point of view of the vector objective function (4).

GAs have the following advantages $(+)$ and disadvantages (-) [2]:

+ no information about the objective function is required;

+ gaps existing on the response surface have little effect on the optimization efficiency;

+ relative resistance to falling into local optima;

+ suitability for solving a wide range of large-scale tasks;

- it is problematic to find an exact global optimum;

- difficult to apply to isolated functions;

- ineffective to apply to optimize a function that takes a long time to calculate;

- it is not possible to find the optimal coding of parameters for all tasks.

When describing the GA, definitions borrowed from genetics are used. 
A population is a finite multitude of individuals. The individuals belonging to the population in the algorithm are determined by a genotype with the multitude of parameters of the problem coded in it. A genotype is a set of chromosomes of a given individual. A chromosome is an ordered sequence of genes. A gene is an atomic element of a genotype, in particular, a chromosome. Locus is a multitude of gene positions. An adaptability function (fitness function, assessment function) is a function that determines the degree of adjustment of a given individual in the population.

Let us examine the use of GA to solve the problem of optimizing the process of component-wise TK of latent failures of a multicomponent PS.

Let us consider an $\mathrm{N}$-component system with a component-wise TCM. The individual in this case will be the control frequency management plan. The vector of times $\bar{\tau}$ determining the control frequency of each of the $n$ components is a characteristic of the individual a genotype. The genotype consists of $N$ chromosomes. The $I$ chromosome determines the control frequency of the $i$ component. As a fitness function to assess the suitability of the individual the objective three-criteria function is used (4).

Each chromosome can be represented as a binary code, where the number of bits $k_{i}$ is determined by the number of possible values of waiting times for monitoring $\tau_{i}$. It is obvious, that the genotype is also represented as a binary code with a bit depth $k=\sum_{i \in 1, N} k_{i}$.

In the genome $k_{1}$ of the lower orders is formed the first chromosome, in the next $k_{2}$ of the orders, the second chromosome, etc. The structure of an arbitrary genome is shown in Figure 3.

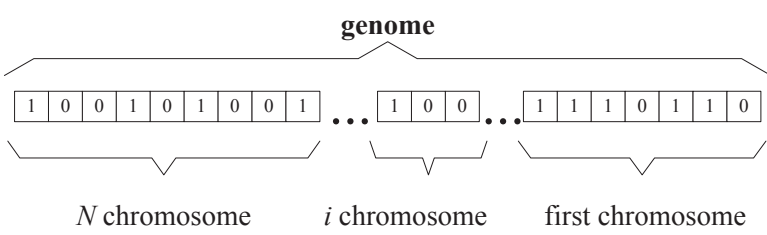

Fig. 3. The genome structure.

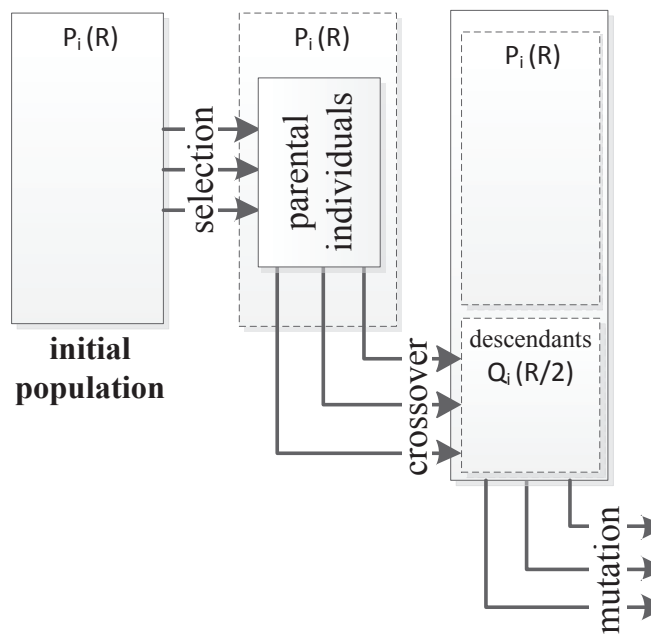

Taking into account the specifics of the problem, the GA with domination sorting and elite selection was chosen (NSGA-II) [13, 14].

Let us describe the work of the chosen GA step by step, taking into account the selected genetic operators (crossover operators, mutations, population generations) and their parameters:

1. After the start of the algorithm, a population of $\mathrm{R}$ individuals is created. The initial population is randomly generated using a uniform distribution.

2. The selection is carried out with the help of the pair tournament selection method.

3. A cross-breeding operation is performed for the selected individuals (parents). Creation of the descendants occurs using a homogeneous crossover. In it, the cross-breeding is performed on the basis of a binary vector, the length of which equals the length of the genome in the locus. Each position of the vector determines the mutual replacement of the locus of the parental individuals. As a result of cross-breeding, the descendants $\mathrm{R} / 2$ are created.

4. The missing part of the individuals $(\mathrm{R} / 2)$ up to twice the population size $(2 * \mathrm{R})$ is formed due to a mutation of already obtained individuals.

5. The individuals are assessed on the level of adaptability. $\mathrm{R}$ of the best adapted individuals are included in the new generation, the others are eliminated. 6 . The steps $2-5$ are repeated, if the exit condition has not been reached.

The block diagram of the used GA is shown in Figure 4 . The algorithm stopping condition is invariance of the best individuals for 50 generations or the achievement of the critical size of the generation's number (500). A high proportion of the mutation of individuals and the applied exit criterion make it possible to reduce the probability of finding a local optimum of the system. At the same time, the use of NSGA-II and the selection tournament allows to accelerate the process of global optimum allocation.

At the end of the algorithm, among the resulting $\mathrm{R}$ individuals, unique ones are selected that make up a multitude that is close to the Pareto set for the set optimization problem.

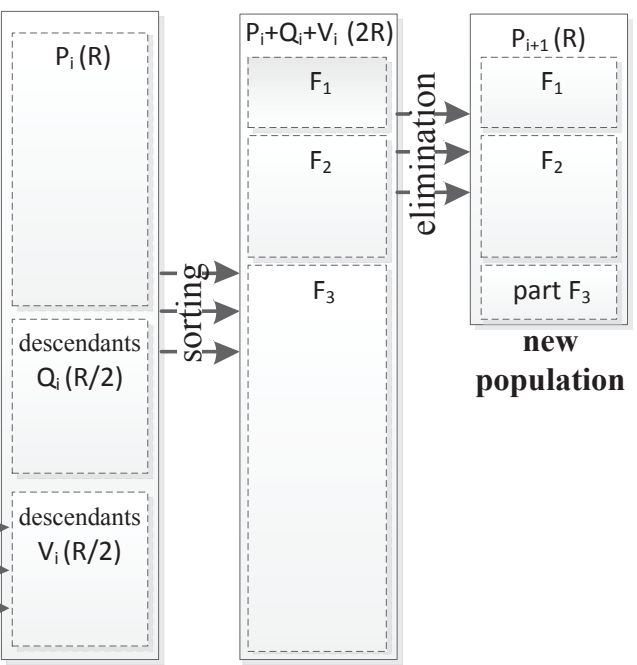

Fig. 4. Block diagram of the used GA. 


\section{Example of ADMS usage}

Let us consider the possibilities of ADMS when solving the problem of optimizing the monitoring frequency of the flux density, the solder quality and the temperature parameters of the system at the WS330-SF Essemtec wave soldering unit [15].

Three TCM are distinguished in the operating PS: flux density monitoring, solder quality monitoring and temperature parameters monitoring. The flux density is monitored every 1.5 hours; in accordance with monitoring results the flux is replaced and a subsequent correction of its density takes place. The solder quality is checked irregularly, on average once every 8 months. The temperature parameters are regulated without carrying out the TCM, if solder defects have been detected. At a wave soldering unit, the soldering of products is carried out in one shift on average for 3 hours per day. In this mode of operation, the percentage of brazed solder joints is $10.2 \%$ of the total number of soldered joints after the installation.

In these conditions, ADMS allows to automate the decision making process; and the decisions are made on the basis of quantitative assessments of the PS technical and economic performance.

In order to solve the problem of "Optimization of component-wise monitoring frequency", the RV parameters determined by the DSS block were used as the initial statistical data to determine the distribution law on the basis of passive production experiment data [16] and obtained as a result of an expert evaluation for the preheating block.

For each monitoring, the following is defined: the minimum monitoring time period, the minimum time step, that the performer can provide. Based on these data, the code length for each of the chromosomes and for the genome, which unambiguously describes the chosen tactics of conducting the component-wise monitoring, is determined.

For the case under consideration, within the constraints imposed, $\sim 8.3$ million (223) of different decisions are possible with respect to the monitoring frequency. The calculation of three system efficiency criteria for one monitoring tactic takes an average of 0.21 second on a PC (2.66 GHz, 4 GB of RAM). To compare, a full calculation of an area close to the Pareto border using the GA (5 populations of 100 individuals), takes 90 seconds on an identical PC. As a result of using the GA, 32 different solutions were obtained.

The objective function values at the Pareto boundary are achieved with the following values of the input parameters (monitoring frequency):

- the monitoring frequency of the fluxing agent varies within the limits of 46 - 50 minutes;

- the monitoring of temperature parameters in view of its high speed and low cost should be carried out continuously, or daily;

- the optimal frequency of the solder quality monitoring varies from continuous to up to 13 days in between monitoring.
At the same time, depending on the chosen strategy, the reduction in the percentage of brazed joints down to $6.3-3.2 \%$ is predicted.

\section{Conclusion}

The ADMS structure was developed and described. The ADMS building concept is based on a hierarchical principle. Two modules of the decision support system were developed: the DSS module for choosing the RV distribution law and/or its parameters, the DSS module for the selection of the TCM optimal frequency. The problem of vector and scalar optimization was posed. To find a multitude close to the Pareto set for multi-criteria optimization of the TCM frequency of the PS with LF, the GA with sorting by domination and elite selection was chosen. To reduce the vector objective function to a scalar one, one of many known methods can be used, for example, the weighting coefficients method.

The research was carried out under the financial support of RFBR within the scope of the scientific project No 15-0105840 .

\section{References}

1. A.V. Lotov, I.I. Pospelova, Multi-criteria Decisionmaking Tasks (2008)

2. T.V. Panchenko, Genetic Algorithms (2007)

3. V.V. Frolov, Eastern European Journal of Advanced Technology 3/9, 60-65 (2012)

4. V. S. Koroluk, Stochastic System Models (1993)

5. Yu.E. Obzherin, E.G. Boyko Semi-Markov Models. Control of Restorable Systems with Latent Failures. (2015)

6. V.I. Denisov, Applied Statistics. The Rules for Verifying Agreement of the Experimental Distribution with the Theoretical One, 1 (1998)

7. B.Yu. Lemeshko, Reliability and Quality Control 11, 3-17 (1997)

8. F. Baykhelt, P. Franken, Reliability and Maintenance. Mathematical Approach (1988)

9. V.S. Avduyevskiy, Reliability and Efficiency in Engineering, 1 (1986)

10. Yu. Obzherin, A. Nikishenko, European International Journal of Science and Technology, Vol. 2 No. 6, 143-152 (2013)

11. A.I. Peschanskiy, A.I. Kovalenko, Automat. and telemech. 12, 112-126 (2016)

12. Ye.G. Boyko, Automation of Processes and Control: Newsletter SevSTU 108, 52-56 (2010)

13. K. Deb, S. Agrawal, A. Pratap, T. NSGA-II. IEEE Transactions on Evolutionary Computation 6, 182197 (2002)

14. Deb, Kalyanmoy, Multi-Objective Optimization Using Evolutionary Algorithms (2001)

15. WS 330-SF. Wave Soldering System. Installation and User Manual — Essemtec, (2007)

16. A.N. Nikishenko, Automation of Processes and Control: Newsletter SevSTU 146, 68-76 (2014) 